\title{
Abnormal Magnetic Resonance Imaging and Hemichorea Associated With Non-Ketotic Hyperglycemia
}

\author{
Hussam A. Yacoub a, b
}

\begin{abstract}
Unilateral basal ganglia abnormalities on brain imaging secondary to non-ketotic hyperglycemia have been reported in the literature with different clinical manifestations. We present two cases of nonketotic hyperglycemia, acute chorea, and reversible CT and MRI findings. There seems to be a wide spectrum of pathological mechanisms underlying this entity, but none of the studies investigating the potential mechanisms of the radiological and clinical presentations have been conclusive.
\end{abstract}

Keywords: Non-ketotic hyperglycemia; Hemichorea

\section{Introduction}

Unilateral basal ganglia computed tomography (CT) and magnetic resonance imaging (MRI) abnormalities secondary to non-ketotic hyperglycemia have been reported in the literature with different clinical manifestations, one of which is a new-onset chorea $[1,2]$. Most cases are diagnosed in diabetic patients, and the syndrome has been reported as the presenting symptom(s) of new-onset diabetes. A triad of non-ketotic hyperglycemia, acute chorea, and a hyperdense and hyperintense putamin on CT and T1-MRI, respectively, is a common presentation. There are usually no signs of mass effect, edema, or volume loss. This entity can mimic ischemic stroke both clinically and radiographically. The abnormal signal on MRI can be unilateral, causing contralateral body symptoms, or bilateral.

Manuscript accepted for publication September 25, 2013

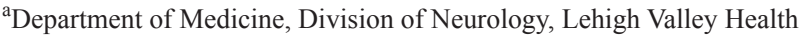
Network, Allentown, Pennsylvania, USA

${ }^{b}$ Corresponding author: Hussam A. Yacoub, 1250 South Cedar Crest

Blvd, Suite 405, Allentown, PA 18103, USA.

Email: hyacoub123@yahoo.com

doi: http://dx.doi.org/10.4021/jnr233e
The pathophysiological mechanism(s) leading to abnormal movements and the described imaging abnormalities in patients with non-ketotic hyperglycemia are not exactly understood. None of the studies investigating the potential mechanism(s) of the radiological and clinical presentations have been conclusive.

We present two cases of non-ketotic hyperglycemia, acute chorea, and reversible CT and MRI findings. We also present cortical enhancement in the right insula and temporal and frontal opercula in this setting, an imaging finding not previously reported.

\section{Case Report}

\section{Case 1}

A 21-year-old man presented with intermittent jerks and occasional writhing of the left arm, along with headache, nausea, and fatigue. He was admitted to the hospital after being found unconscious.

The patient had a history of insulin-dependent diabetes and seizures since age 14. Family history was significant for hypertension and diabetes. Home medications included lantus 35 units at bedtime and one unit novolog per $15 \mathrm{~g}$ of carbohydrates with each meal. He was non-compliant with his medications. He smoked one pack of cigarettes per day and drank alcohol occasionally.

He was afebrile with a blood pressure of 140/80, pulse of 88 , and respiration rate of 18 . On neurological examination, he was found to be lethargic but arousable to verbal stimuli. He opened his eyes with poor eye contact. He was oriented to place, person, and time. Intermittent choreoathetoid movements of the left shoulder were noted, as well as occasional myoclonic jerks of the left hand. Motor examination revealed a drift in the left arm. Reflexes were decreased throughout. He had mild ataxia on finger-to-nose testing on the left.

The patient had a witnessed generalized tonic-clonic seizure in the emergency room. Serum glucose level was too high to be registered. Laboratory work-up revealed a WBC of $17,900 / \mu \mathrm{L}$, platelets $703,000 / \mu \mathrm{L}$, sodium $136 \mathrm{mEq} / \mathrm{L}$, po- 

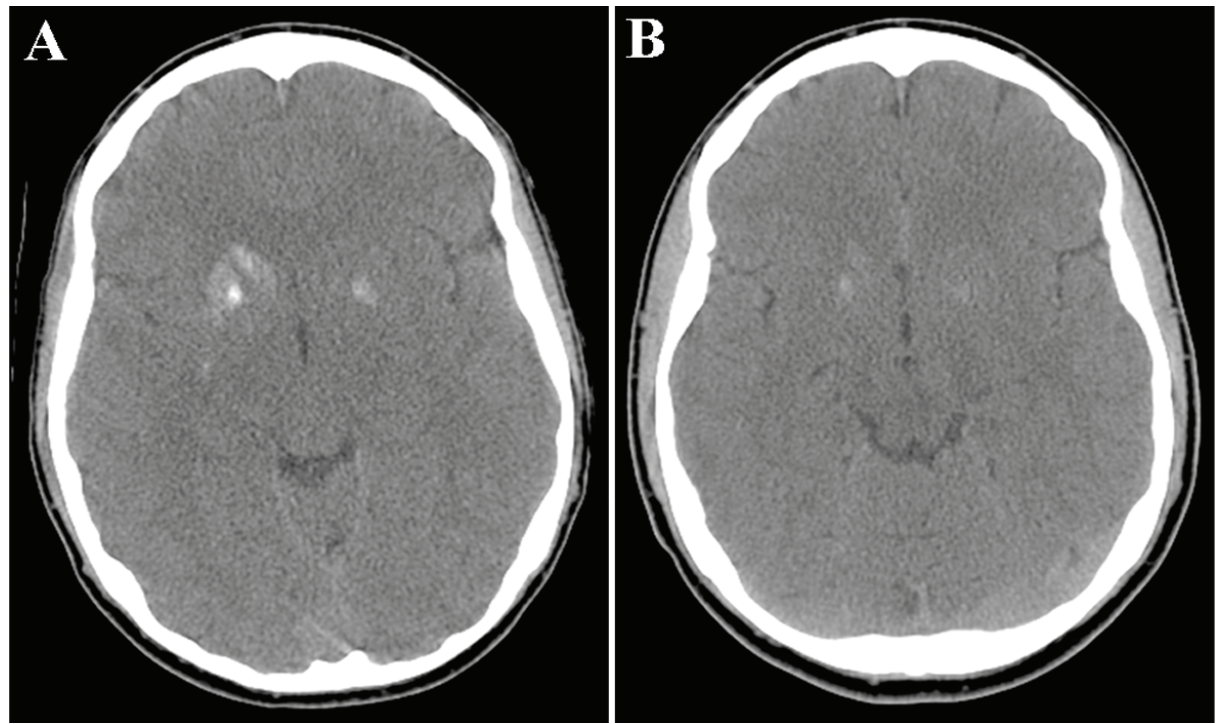

Figure 1. (A) A CT of the head without contrast demonstrated an area of hyperdensity involving the right head of caudate, putamen, and globus pallidus, and to a lesser extent, the left putamen, with no surrounding edema. (B) Partial resolution of the increased density in the right basal ganglia.

tassium $6.0 \mathrm{mEq} / \mathrm{L}$, carbon dioxide $<5 \mathrm{mmol} / \mathrm{L}$, and serum glucose $644 \mathrm{mg} / \mathrm{dL}$.

A CT of the head showed an area of hyperdensity involving the right head of caudate, putamen, and globus pallidus, and to a lesser extent, the left putamen, with no surrounding edema or mass effect (Fig. 1A). An MRI of the brain showed an increased signal in the head of caudate and putamen on the right, and to a lesser extent, on the left, on T1-sequence (Fig. 2A), corresponding to a hypointensity on T2 sequence (data not shown). There was no pathological enhancement or restricted diffusion. An electroencephalogram was unremarkable.

The patient was admitted for management of diabetic ketoacidosis and started on the insulin drip. The abnormal movements of the right arm significantly improved and he was discharged home.

A repeat CT of the head 6 months later showed smaller areas of increased density in the right basal ganglia when
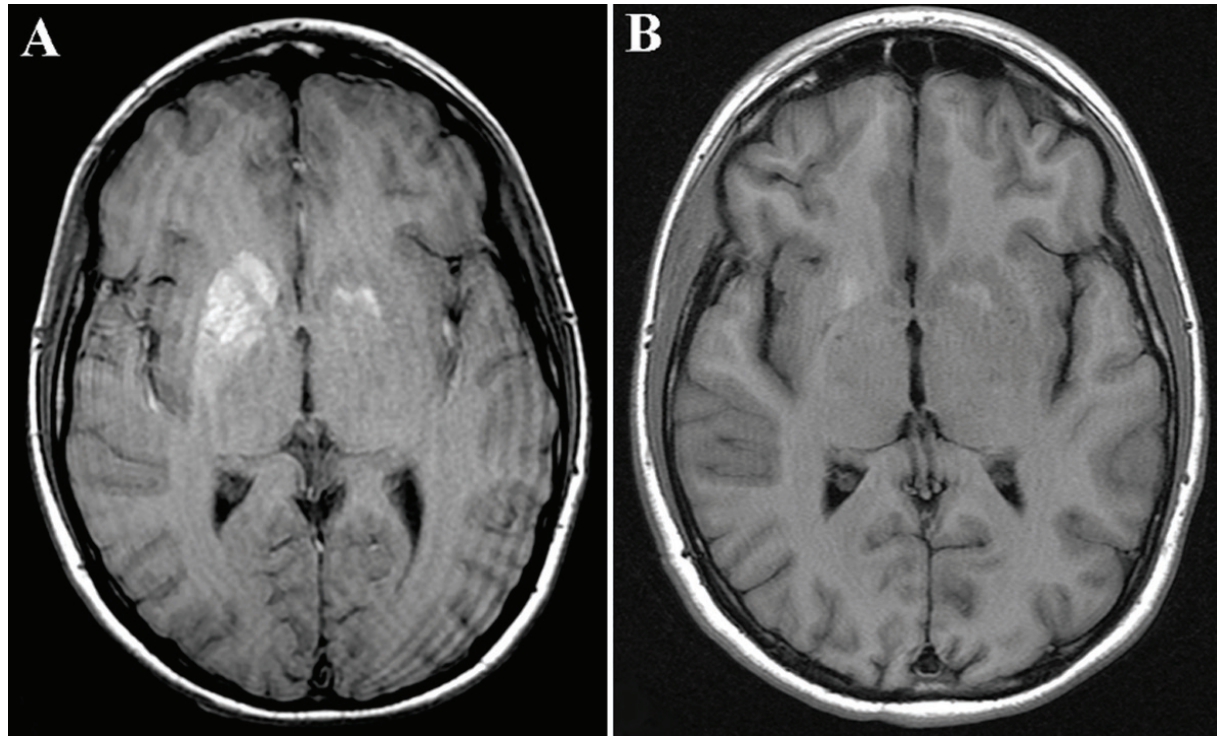

Figure 2. (A) MRI T1-sequence of the brain showed an increased signal in the head of caudate and putamen on the right, and to a lesser extent, on the left. (B) Partial resolution of the increased signal abnormality in bilateral basal ganglia. 


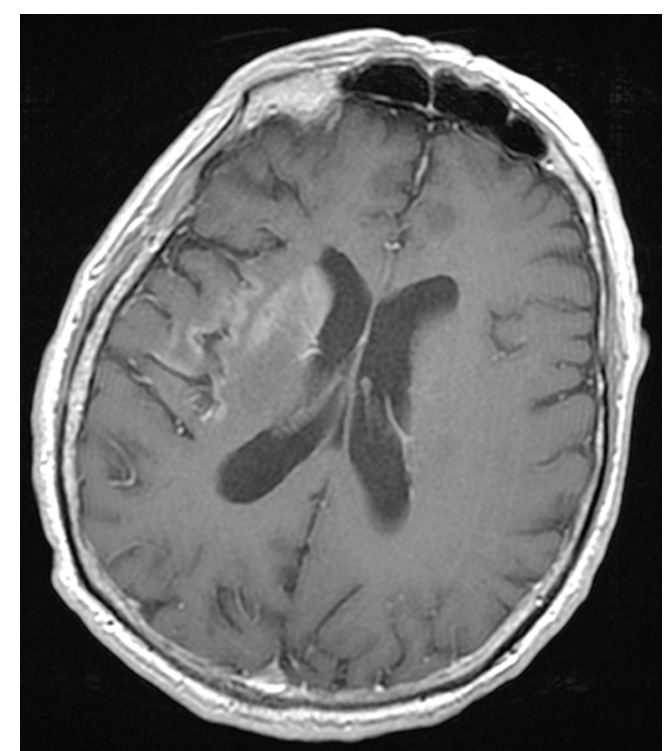

Figure 3. An MRI of the brain with gadolinium revealed cortical enhancement in the right insula.

compared with the previous scan (Fig. 1B). A repeat MRI 14 weeks later showed partial resolution of the increased signal in the basal ganglia on $\mathrm{T} 1$ sequence (Fig. 2B).

\section{Case 2}

A 72-year-old man presented with mild confusion and leftsided weakness for 4 days, along with fatigue and intermittent right-sided frontal throbbing headache for a week. He also had difficulty speaking and ambulating.

The patient had no known history of diabetes. He smoked one-half pack of cigarettes per day and admitted to heavy alcohol use until 2 years prior. He was not on any prescribed medications.

He was afebrile with a blood pressure of 164/74 and a heart rate of 64. On neurological examination, he was found to be awake, alert, and oriented to person, time, and place. He was not dysarthric or aphasic. Cranial nerve, sensory, motor, and coordination examinations were unremarkable. Reflexes were decreased in both lower extremities. Laboratory workup revealed a $\mathrm{WBC}$ of $6,300 / \mu \mathrm{L}$, platelets $159,000 / \mu \mathrm{L}$, sodium $141 \mathrm{mEq} / \mathrm{L}$, potassium $2.7 \mathrm{mEq} / \mathrm{L}$, carbon dioxide 29 $\mathrm{mmol} / \mathrm{L}$, and serum glucose $613 \mathrm{mg} / \mathrm{dL}$.

A CT of the head showed an area of hyperdensity involving the right head of caudate, putamen, insula, and cerebral peduncle with no surrounding edema or mass effect (data not shown). An MRI of the brain showed an increased signal in the right caudate and basal ganglia on $\mathrm{T} 1$ sequence, areas that corresponded to the hyperdense regions seen on the CT, without pathological enhancement or restricted diffusion. The MRI also revealed cortical enhancement in the right insula and temporal and frontal opercula with mild T2 but not T1-hyperintensity (Fig. 3). These radiographic findings were initially considered a result of subacute ischemia with hemorrhagic transformation in the right basal ganglia and caudate.

The patient's symptoms improved as the hyperglycemia was appropriately managed and he was discharged in a stable condition. He returned to our hospital 2 weeks later with painful, writhing movements of the left hand followed by occasional high amplitude movements of the entire left arm. A CT of the head demonstrated partial resolution of the previously noted hyperdensity in the right head of caudate and basal ganglia. He was started on levetiracetam and discharged home. A CT of the head repeated 7 months later revealed partial resolution of the increased density in the right basal ganglia.

\section{Discussion}

Pathophysiological mechanism(s) leading to the described imaging abnormalities in patients with non-ketotic hyperglycemia are not well understood. Some studies report myelin destruction and the accumulation of myelin breakdown products as a potential mechanism [3], whereas other authors contribute it to hyperviscosity [2].

In both our patients, there was clinical improvement on follow-up as well as radiographic evidence of resolution of the abnormal findings seen on initial studies. This, along with other reports in the literature [4], suggests that the mechanism(s) responsible for non-ketotic hyperglycemic radiographic and clinical manifestations is a reversible insult in the setting of marked hyperglycemia.

Studies utilizing radiological findings have been uncertain in identifying the pathological mechanisms associated with non-ketotic hyperglycemia. Putaminal petechial hemorrhage has been proposed as a potential mechanism underlying the abnormal MRI signal [5]. Blood-brain barrier destruction as a result of hyperglycemia and consequent erythrocyte diapedesis has been proposed as a potential cause of petechial microhemorrhage in the putamen [6]. Wintermark et al [7] reported a case of a patient with hyperglycemia-induced chorea and areas of restricted diffusion in the contralateral putamin on MRI. A two-case series of hyperglycemic hemichorea revealed restricted diffusion on diffusion-weighted imaging (DWI) and apparent diffusion coefficient (ADC) which suggests cytotoxic edema as a potential mechanism [2]. Ischemia, but not hemorrhage or calcification, may account for restricted diffusion findings on DWI and ADC. The hyperattenuation observed a month later, as well as the lack of restricted diffusion in both our cases, argues against ischemia as a potential mechanism associated with neurological and radiographic findings of non-ketotic hyperglycemia.

Abnormal deposition of calcium in neurons has been 
also proposed as a potential pathological mechanism explaining the CT and MRI changes associated with non-ketotic hyperglycemia [7]. Complete or partial resolution of the basal ganglia hyperintensity, as was seen in both our cases, refutes this theory and suggests an alternative mechanism(s).

In the majority of reported cases in the literature, imaging studies reveal evidence of a hyperdensity on CT scan corresponding to hyperintensity and hypointensity on T1and T2-weighted MRI, respectively [8]. In case 2 above, an interesting finding of insular cortex enhancement was also observed. To our knowledge, this finding has not been previously reported in the literature in the setting of non-ketotic hyperglycemia. The mechanism(s) explaining this phenomenon remains unclear.

Hemichorea associated with non-ketotic hyperglycemia and abnormal MRI signals have been frequently reported in Asian, especially elderly, women with diabetes mellitus type II. The two cases of non-ketotic hyperglycemia associated with abnormal MRI signal reported by Wintermark et al [7] and in 30 out of 35 cases analyzed by Lin et al [9] were described in Asian patients, suggesting a genetic disposition. Interestingly, neither of our patients was of an Asian descent.

It is imperative to recognize unilateral basal ganglia hyperdensity on CT and abnormal signal on MRI, occasionally associated with restricted diffusion, in patients with nonketotic hyperglycemia. The radiographic findings and the clinical presentation can mimic stroke; therefore, it is vital to distinguish the two entities, as patient management and clinical outcome may vary greatly. The prognosis of the abnormal movements appears excellent, and the radiological abnormalities seem reversible.

In summary, none of the studies investigating the potential mechanisms of the radiological and clinical presentations associated with non-ketotic hyperglycemia have been definite. There appears to be a wide spectrum of pathological mechanisms underlying this entity. Cytotoxic edema, microhemorrhages, myelin breakdown, hyperviscosity, and ischemia have all been proposed as potential mechanisms, but none of the studies are completely persuasive. The nature of the brain imaging findings associated with non-ketotic hyperglycemia remains a subject of controversy.

\section{Acknowledgement}

The author wishes to acknowledge the generous contributions by Jacqueline Grove, Medical Editor, Lehigh Valley Health Network, in the review and preparation of this manuscript.

\section{Conflicts of Interest}

The author declares no conflicts of interest.

\section{References}

1. Oh SH, Lee KY, Im JH, Lee MS. Chorea associated with non-ketotic hyperglycemia and hyperintensity basal ganglia lesion on T1-weighted brain MRI study: a metaanalysis of 53 cases including four present cases. J Neurol Sci. 2002;200(1-2):57-62.

2. Chu K, Kang DW, Kim DE, Park SH, Roh JK. Diffusion-weighted and gradient echo magnetic resonance findings of hemichorea-hemiballismus associated with diabetic hyperglycemia: a hyperviscosity syndrome? Arch Neurol. 2002;59(3):448-452.

3. Chang MH, Chiang HT, Lai PH, Sy CG, Lee SS, Lo YY. Putaminal petechial haemorrhage as the cause of chorea: a neuroimaging study. J Neurol Neurosurg Psychiatry. 1997;63(3):300-303.

4. Chang KH, Tsou JC, Chen ST, Ro LS, Lyu RK, Chang HS, Hsu WC, et al. Temporal features of magnetic resonance imaging and spectroscopy in non-ketotic hyperglycemic chorea-ballism patients. Eur J Neurol. 2010;17(4):589-593.

5. Mestre TA, Ferreira JJ, Pimentel J. Putaminal petechial haemorrhage as the cause of non-ketotic hyperglycaemic chorea: a neuropathological case correlated with MRI findings. J Neurol Neurosurg Psychiatry. 2007;78(5):549-550

6. Iwata A, Koike F, Arasaki K, Tamaki M. Blood brain barrier destruction in hyperglycemic chorea in a patient with poorly controlled diabetes. J Neurol Sci. 1999;163(1):90-93.

7. Wintermark M, Fischbein NJ, Mukherjee P, Yuh EL, Dillon WP. Unilateral putaminal CT, MR, and diffusion abnormalities secondary to nonketotic hyperglycemia in the setting of acute neurologic symptoms mimicking stroke. AJNR Am J Neuroradiol. 2004;25(6):975-976.

8. Lai PH, Tien RD, Chang MH, Teng MM, Yang CF, Pan $\mathrm{HB}$, Chen C, et al. Chorea-ballismus with nonketotic hyperglycemia in primary diabetes mellitus. AJNR Am J Neuroradiol. 1996;17(6):1057-1064.

9. Lin JJ, Lin GY, Shih C, Shen WC. Presentation of striatal hyperintensity on T1-weighted MRI in patients with hemiballism-hemichorea caused by non-ketotic hyperglycemia: report of seven new cases and a review of literature. J Neurol. 2001;248(9):750-755. 